\title{
Commentaires dans
la Presse Quotidienne
Régionale française en ligne
et politisation du local
}

\author{
Franck Bousquet \\ LERASS \\ Université Toulouse-3 Paul Sabatier \\ France \\ franck.bousquet@iut-tlse3.fr
}

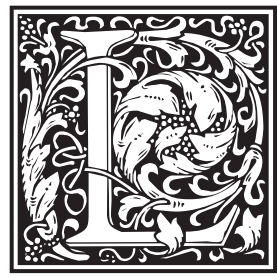

es contraintes qui président à la fabrication et à la transmission de l'information locale et régionale sont nombreuses et tendent à se multiplier. D'abord, le lien de dépendance séculaire de la plupart des journaux infranationaux envers les autorités de toutes sortes prend des formes nouvelles et plus contraignantes depuis l'avènement de la communication généralisée et avec la professionnalisation de tous les acteurs, publics et privés, en charge de sa mise en oeuvre (Miège,1990 ; Frisque, 2010). Ensuite, la fragilité économique des entreprises d'informations entraîne la réduction du nombre de journalistes et la dépendance accrue envers les annonceurs (Bousquet et al., 2015). Enfin, les bouleversements importants concomitants au passage en ligne d'une partie de l'activité journalistique et de sa transmission, créent de nouveaux carcans et de nouvelles obligations pour les professionnels de l'information (Estienne, 2007).

Ainsi, il apparaît que le lien entre l'information et l'animation d'un débat public territorialisé (Le Bohec, 2000) ne constitue pas un axe prioritaire pour les entreprises de presse locales traditionnelles, qui préfèrent mettre en avant le journalisme de solution (Amiel, 2017), l'information service ou le développement du territoire ; directions qu'elles considèrent plus rentables. Pourtant, sur bien des territoires, le journal régional est le seul acteur professionnel à produire une information quotidienne complète

Pour citer cet article, to quote this article, para citar este artigo :

Franck Bousquet, «Commentaires dans la Presse Quotidienne Régionale française en ligne et politisation du local », Sur le journalisme, About journalism, Sobre jornalismo [En ligne, online], Vol 7, n² 2 - 2018, 15 décembre - December 15 -

15 de dezembro.

URL : http://www.surlejournalisme.com/rev 
et devrait donc, selon des auteurs comme Dewey, jouer un rôle primordial dans la construction des publics et des sujets qui les animent (1927).

La notion de public, telle qu'abordée dans le débat entre Lippmann (1925) et Dewey (1927), nous semble en effet être une porte d'entrée intéressante pour traiter de la place de l'information locale dans le débat démocratique car elle rend possible l'analyse du travail journalistique en tant qu'acteur participant à la définition des principaux enjeux qui président à la constitution de communautés de sens territorialement situées. Elle peut aussi rendre compte de la façon dont les sujets politiques se forment au niveau local. Ces questions, traitées à travers l'étude de médias ou de groupes Facebook nationaux (Servais, 2017) sont systématiquement ignorées pour les arènes locales. Pourtant, la politisation du local, à savoir l'articulation entre des questions territoriales et des valeurs de portée générale incarnées par des groupes politiques, a été une question majeure pour l'établissement du suffrage universel (Agulhon, 1970) et pour l'enracinement de la République en France (De La Haye, 1984). C'est un sujet désormais abordé par l'ethnologie politique (Vignon, 2016) mais la dimension médiatique est curieusement absente dans ce type d'études.

Ainsi, comme le souligne Dewey "seule une interprétation inlassable et quotidienne "des nouvelles " " peut permettre la constitution d'un public, entendu comme un ensemble d'individus d'accord sur un ensemble de questions nécessitant discussion et traitement politique (1927, p.280). Le rôle des médias d'informations, y compris à l'échelle d'une commune moyenne ${ }^{1}$, est par conséquent primordial quant à la création de ces communautés d’interprétation.

Les sujets qui nous intéresseront sont par conséquent d'ordre politique, entendus comme tels à partir du moment où ils incluent

« la référence aux principes généraux devant régir une société ou, dans les termes de Boltanski, la montée en généralité, et la reconnaissance de la dimension conflictuelle des positions adoptées (au sens où le locuteur admet l'existence de clivages sur la question en jeu, et non pas au sens où il mobiliserait nécessairement un registre discursif revendicatif ou contestataire)» (Hamidi, 2006).

Aujourd'hui, les commentaires sur les sites de presse sont parmi les moyens d'expression les plus populaires auprès des internautes, même si le dispositif socio-technique employé les contraint et les rend peu visibles. Certains auteurs font même l'hy- pothèse qu'ils participent à modeler le journalisme et qu'ils ont un impact sur la sphère publique (Graham, Wright, 2015), en particulier en remettant en question les routines journalistiques (Hermida, Thurman, 2008). Pourtant, les journalistes, selon la littérature, décrivent souvent les commentaires comme agressifs, de piètre qualité, faux, non représentatifs et déplorent également qu’ils émanent de lecteurs ne payant pas pour leur travail (Bergström, Wadbring 2014 ; Aubert, 2011). Certains médias en viennent même à faire disparaître la section commentaire ou à la rendre de plus en plus difficile d'accès et de moins en moins visible, favorisant de la sorte le déplacement du type d'expression qu'elle contenait vers les réseaux socio-numériques (Jouët, Le Caroff, 2013).

C'est cependant à travers l'étude des commentaires postés à la suite des articles d'un journal de Presse Quotidienne Régionale (PQR) consacrés à une petite commune française que nous voudrions tester la formation de publics locaux. Cela nous permettra de territorialiser des questionnements aujourd'hui la plupart du temps réservés à la presse nationale (Hermida et al., 2011) et de questionner la PQR par rapport à un rôle qui a été fondateur pour elle (De La Haye, 1984). Nous nous interrogerons en effet sur le type de rassemblement que ce dispositif rend possible, sur les caractéristiques de ce que les internautes partagent par son intermédiaire ainsi que sur l'influence de l'orientation éditoriale du titre de presse dans ces processus.

\section{TERRAIN ET MÉTHODOLOGIE}

Le territoire choisi pour l'étude est celui de la commune de Moissac, ville de 13000 habitants, incluse dans une intercommunalité de 40000 personnes et située à $80 \mathrm{~km}$ de Toulouse, dans le Tarn-et-Garonne, département du Sud-Ouest de la France. La période d'étude est celle des élections municipales de 2014. Il nous semble en effet qu'une période d'élections locales est particulièrement propice pour la formulation d'opinions politiques et pour l'échange d'arguments. Notre objectif étant d'observer l'existence ou la formation d'éventuels publics politiques locaux, les élections municipales, élections locales à la plus forte participation, sont par conséquent idoines.

D’un point de vue politique, Moissac a alors un maire socialiste et deux conseillers généraux Radical de Gauche. Maire depuis 1983, Jean-Paul Nunzi se re-présente pour un sixième mandat. Député socialiste à 2 reprises, il n'est, à ce moment-là, plus membre du parti mais continue à en incarner symboliquement la représentation locale. Trois listes se 
présentent contre celle dont il occupe la première place.

L'une est menée par son ancien adjoint radical, démis de ses fonctions 8 mois auparavant, par ailleurs conseiller général et soutien de Jean-Michel Baylet, président du conseil général depuis 1985 et directeur de La Dépêche du Midi (voir infra). Cette liste comprend aussi le second conseiller général de la ville, ancien rédacteur en chef de La Dépêche $d u$ Midi. Elle se présente elle aussi sans étiquette apparente, même si son affiliation au Parti Radical de Gauche est largement publique.

La deuxième est une liste «Union de la droite », menée par un médecin retraité.

La troisième, avec l'étiquette "Rassemblement Bleu Marine », a comme tête de liste un ex-membre du principal parti conservateur (UMP) ayant rejoint le Front National.

La Dépêche du Midi est le seul média à construire une information journalistique quotidienne pour cette commune. Ce titre est présent sur 10 départements et fait partie d'un groupe de presse monopolistique en Occitanie puisque possédant également Le Midi Libre et L'Indépendant. Son PDG est Jean-Michel Baylet, héritier du capital politique et médiatique familial, président du Parti Radical de Gauche de 1995 à 2015, ministre ou secrétaire d'État dans les gouvernements Fabius, Rocard, Cresson, Bérégovoy et Valls, et parlementaire (député puis sénateur) depuis 1976. Le Tarn-et-Garonne, et plus encore la seconde circonscription législative de ce département, est le berceau du pouvoir familial des Baylet et leur fief électoral.

Cette situation est à la fois ordinaire et extraordinaire dans le contexte français. Ordinaire car désormais des groupes de presse de plus en plus gros se partagent le territoire de l'information infra-nationale. Ils sont chacun monopolistique sur des territoires de plus en plus importants. Extraordinaire car La Dépêche du Midi est le seul titre dont le propriétaire est un homme politique de premier plan qui mélange de façon systématique son activité politique et la ligne éditoriale de son journal.

Intégralement en ligne gratuitement depuis 2006, La Dépêche permet aux internautes de poster des commentaires à condition qu'ils enregistrent un compte. Les seules données indispensables pour s'inscrire sont une adresse électronique valable, l'indication de la commune de résidence et le choix d'un pseudonyme.
La modération du site a longtemps été effectuée en deux temps. D’abord une modération automatique et a priori bannissait les commentaires comportant certains mots clés avant qu'une intervention humaine n'intervienne. Toutes les insultes et les expressions ordurières sont interdites mais aussi certains noms propres comme celui du PDG du journal ou de ses proches. Aucun commentaire comportant les noms « Baylet » ou « Pinel $^{2}$ » ne peut par exemple être publié. La découverte de cette interdiction, pour le moins singulière, résulte d'une observation des commentaires et nous a été confirmée lors d'un entretien dans les locaux de Ladepeche.fr par la responsable de la modération. Elle serait justifiée par la violence régulière qui s'exprime dans les commentaires à l'encontre de ces deux figures majeures de la politique locale. Une charte de modération est en outre disponible sur le site. Elle n'affiche que des principes généraux (interdiction des allusions sexistes, homophobes et racistes, des messages publicitaires, des petites annonces, des liens externes, de la diffamation, de l'insulte...). Depuis 2013, donc au moment de notre étude, le marché de la modération a été confié à la société Atchik (Smyrnaios, Marty, 2017) qui a pour mission de vérifier a priori que cette charte soit bien respectée ${ }^{3}$. Une modération interne est cependant toujours possible car le contrôle final de l'édition appartient à Ladepeche.fr.

Pour notre étude, nous avons sélectionné 93 articles sur les 114 publiés consacrés à la commune sur le site du journal régional (tous ceux qui ont été commentés, quel que soit leur sujet) et les 528 contributions des 134 commentateurs qu'ils ont suscitées, sur la période allant du $1^{\mathrm{er}}$ janvier 2014 au 23 mars 2014, que nous avons considérée comme étant celle où la campagne électorale des municipales devenait visible.

Nous avons classé les articles selon leurs thèmes et leurs éventuelles prises de position, directe ou indirecte, dans la campagne. Il nous est ainsi apparu que 6 grandes catégories d'articles pouvaient être repérées : vie municipale (articles sur les conseils municipaux, les décisions des élus et leurs conséquences ainsi que la campagne pour les élections) ; vie associative (articles sur les activités du monde associatif) ; vie économique et sociale (acteurs économiques, solidarité, agriculture, tourisme) ; sport ; commémoration ; faits divers.

Nous avons ensuite comptabilisé les citations et les photographies des différents candidats au sein de chacun des articles du corpus. Cette première étape, indispensable pour analyser les commentaires des lecteurs, nous a permis de poser le contexte du traitement de la campagne, et plus largement de l'actualité de la vie communale. Elle 
nous permettra aussi de déterminer si certains articles sont plus "déclencheurs " que d'autres et d’identifier ce qu'ils "déclenchent " (Dupret, Klauss, Ghazzal, 2010).

Dans un second temps nous avons compté le nombre de commentaires et le nombre de commentateurs par article, identifié les commentaires supprimés et repéré les interactions entre les commentateurs ${ }^{4}$. Par la suite nous avons traité ces commentaires selon une approche qualitative, en nous inscrivant dans une démarche d'analyse de discours (Charaudeau, Maingueneau, 2002).

Nous avons ainsi choisi d'étudier les commentaires en employant des catégories qui résultent de notre questionnement. Nous les avons d'abord classés en fonction de leur positionnement par rapport à la campagne municipale. Sont-ils explicitement ou implicitement partisans ? Quelle forme prend leur prise de position et leur soutien?

Ensuite nous les avons interrogés en fonction de leur position sur la ligne éditoriale du journal. Expriment-ils une opinion à ce sujet ? Laquelle ? Le fontils de façon explicite (en le revendiquant) ou est-ce plus indirect?

Nous avons également tenté de déterminer si les commentaires font un lien entre la situation et les faits locaux décrits dans les articles et un contexte général que l'on pourrait qualifier de politique -selon la définition donnée dans l'introduction- et de quelle façon : explicitement en se référant à des acteurs politiques, des instances partisanes ou des programmes d'actions ; ou implicitement en rapportant des faits à des valeurs ou en mettant en lien des faits dont le rapprochement produit un sens par rapport aux débats politiques contemporains. Enfin, nous avons essayé de déterminer si une interaction peut s'observer entre les commentateurs et quelle forme elle prend : conversation, échange d'arguments, affirmation identitaire, interjection...

Notre objectif est double. D'abord il s'agit de vérifier si des communautés d'interprétation se forment, en s'accordant pour identifier des sujets de discussion donnant une signification politique à des faits ou des situations locales. Ensuite il convient d'évaluer le rôle du journal et de sa production dans ce processus. Est-il initiateur des discussions, partenaire ou prétexte ? Comment les contributeurs se positionnent-ils par rapport à lui ? Est-ce que les enjeux qui président à la création de ces éventuels publics sont les mêmes que ceux développés par les journalistes locaux et les acteurs politiques (Marty, 2015) ?

\section{UN TRAITEMENT MÉdiATIQUE POLITIQUE ET PARTISAN}

Concernant la couverture médiatique de la ville de Moissac entre le 1 er janvier et le 23 mars deux éléments majeurs apparaissent : l'intérêt très fort du journal pour les sujets pouvant donner lieu à des enjeux électoraux et le caractère très partisan de l'orientation éditoriale. En effet, sur les 93 articles analysés sur la période, 54 , c'est-à-dire près de $59 \%$, sont en relation directe ou indirecte avec la campagne électorale ou ses enjeux. 41 présentent un intérêt politique directement assumé, en traitant des sujets en lien avec l'activité municipale et pouvant donner lieu à débat et à prise de position, et 13 présentent un intérêt politique caché en mettant en scène les activités ou les actions de certains candidats à l'élection dans des situations qui n'y sont pas associées. Ce premier résultat est intéressant car il va à l'encontre d'une opinion répandue quant au rôle présumé peu important de la PQR dans l'animation des débats politiques locaux (Ballarini, 2008). La Dépêche du Midi, du moins pour cette commune et lors de cette élection, est un acteur politique majeur.

Cependant, en corrolaire de ce constat, il apparaît que le journal de Jean-Michel Baylet est un acteur très engagé. Il prend ouvertement position pour un candidat et pose l'élection comme un duel entre deux personnes, effaçant de fait les deux autres listes. Cela transparaît directement dans la question que pose un journaliste au candidat PRG dans l'un des entretiens publiés "Dans cette campagne, on a l'impression qu'il n'y a que deux listes, celle du maire sortant et la vôtre " $(22 / 02 / 2014)$, et va totalement à l'encontre des cadrages nationaux qui prédisent partout des scores importants pour la droite et l'extrême droite et qui se vérifieront localement lors du scrutin.

Le soutien en direction de la liste des deux conseillers généraux se traduit de trois façons différentes : par une sur-représentation en termes de citations et de photographies, par une ligne éditoriale du journal calquée sur leurs positions, en particulier celles qui attaquent le maire sortant, et par une tribune ouverte dans les pages locales.

Ainsi, les deux candidats radicaux sont les seuls à bénéficier d'entretiens dans le journal (4 fois sur la période étudiée) et de tribunes publiées (3 fois sur la période). Ils sont aussi les plus représentés en termes de photographies, 30 fois pour la tête de liste et 16 fois pour son colistier (contre 10 photos pour le maire sortant et 1 pour chacun des deux autres candidats). Ils sont en outre les seuls à avoir des photos individuelles, les autres sont toujours au milieu d'autres personnes. Du point de vue des cita- 
tions, Pierre Guillamat (tête de liste) est cité 30 fois, Guy-Michel Empociello (l'autre conseiller général) 18 fois alors que dans le même temps le maire sortant est cité 18 fois et les candidats de droite et d'extrême droite respectivement 2 et 3 fois. Ces articles, même s'ils n'abordent pas directement la campagne, mettent en scène leurs candidatures et témoignent donc d'un intérêt politique caché.

Du point de vue éditorial la prise de position est encore plus évidente. En effet sur les 18 citations impliquant le maire sortant 9 remettent en cause son action (sur le budget, le patrimoine, l'aire d'accueil des gens du voyage, la composition de sa liste...) et 9 signalent sa présence à un événement. Il est en outre à noter que ces 9 citations sont le fait de notules produites par des correspondants. Jamais les journalistes en poste, en charge de la politique éditoriale du journal, ne citent le maire socialiste pour une autre raison que pour une attaque de son action ou de sa politique. Il bénéficie en revanche, de par sa position, d'une présence dans le journal (même si elle est négative). Ce qui n'est pas le cas des candidats UMP et FN qui en sont totalement absents. Notons pour terminer que le résultat de l'élection viendra infirmer l'éditorialisation de la campagne par La Dépêche en voyant le candidat de la droite classique l'emporter.

\section{Les commentateurs}

Sur l'ensemble des 93 articles mis en ligne durant les 3 mois étudiés, 528 commentaires ont été publiés par 132 commentateurs. Cependant, derrière ce chiffre global, se cache une réalité très contrastée, tant du point de vue des commentateurs que des commentaires. Nous avons d'abord identifié 4 grandes classes de commentateurs :

- les commentateurs très assidus, qui ont posté chacun plus de 20 commentaires.

- les commentateurs assidus, qui ont posté entre 10 et 20 commentaires (soit entre 1 et 2 commentaires par semaine).

- les commentateurs occasionnels, qui ont posté entre 5 et 10 commentaires.

- les commentateurs très occasionnels, qui ont posté moins de 5 commentaires.

Il convient de noter que ces chiffres ne concernent que les commentaires postés sous les articles consacrés à la ville de Moissac. Un commentateur très

Tableau de la couverture éditoriale de ville de Moissac durant la campagne municipale par la Dépêche du Midi.

\begin{tabular}{|c|c|c|c|c|c|}
\hline Sujets des articles & Nombre & Candidats cités & $\begin{array}{l}\text { Photos candi- } \\
\text { dats }\end{array}$ & $\begin{array}{l}\text { Intérêt Pol } \\
\text { assumé }\end{array}$ & $\begin{array}{l}\text { Intérêt Pol } \\
\text { caché }\end{array}$ \\
\hline Vie municipale & 32 & $\begin{array}{l}12 \text { PRG } 1 \\
6 \text { PRG } 2 \\
8 \text { PS } \\
3 \text { FN } \\
2 \text { UMP }\end{array}$ & $\begin{array}{l}11 \text { PRG } 1 \\
5 \text { PRG } 2 \\
4 \text { PS } \\
1 \text { UMP } \\
1 \mathrm{FN}\end{array}$ & 32 & 0 \\
\hline $\begin{array}{l}\text { Vie économique et } \\
\text { sociale }\end{array}$ & 14 & $\begin{array}{l}7 \text { PRG } 1 \\
7 \text { PRG } 2 \\
1 \text { PS }\end{array}$ & $\begin{array}{l}8 \text { PRG } 1 \\
7 \text { PRG } 2\end{array}$ & 6 & 4 \\
\hline Vie associative & 21 & $\begin{array}{l}3 \text { PRG } 2 \\
8 \text { PRG } 1 \\
6 \text { PS }\end{array}$ & $\begin{array}{l}10 \text { PRG } 1 \\
4 \text { PRG } 2 \\
6 \text { PS }\end{array}$ & 2 & 6 \\
\hline Sport & 10 & & & 0 & 1 \\
\hline Commémoration & 2 & $\begin{array}{l}2 \text { PRG } 2 \\
1 \text { PRG } 1 \\
1 \text { PS }\end{array}$ & $\begin{array}{l}1 \text { PRG } 3 \text { (JM } \\
\text { Baylet) }\end{array}$ & 0 & 1 \\
\hline Faits Divers & 14 & 2 PRG 1 & 1 PRG 1 & 1 & 1 \\
\hline Total & 93 & $\begin{array}{l}30 \text { PRG } 1 \\
18 \text { PRG } 2 \\
18 \text { PS } \\
3 \mathrm{FN} \\
2 \mathrm{UMP}\end{array}$ & $\begin{array}{l}30 \text { PRG } 1 \\
16 \text { PRG } 2 \\
10 \text { PS } \\
1 \text { UMP } \\
1 \mathrm{FN}\end{array}$ & 41 & 13 \\
\hline
\end{tabular}


occasionnel sur cette page peut être très assidu sur l'ensemble du site du journal.

Dans la première catégorie nous ne retrouvons que 4 commentateurs : Ozaguay avec 84 commentaires ; JD82200 avec 47 commentaires ; NoelledeMoissac avec 42 commentaires et Patrice Charles avec 22 commentaires. Notons que ce dernier n'emploie pas de pseudonyme et qu'il s'agit de la tête de liste Front National aux élections municipales. Dans les autres catégories nous avons 7 commentateurs à plus de 10 commentaires, 5 entre 5 et 10,8 avec 4 commentaires, 7 avec 3,17 avec 2 et enfin 84 personnes n'ont posté qu'un seul commentaire sous les articles consacrés à la ville de Moissac. Ces résultats confortent les études qui démontrent qu une minorité de lecteurs en ligne participent, mais qu'ils sont très actifs (Norris, 2001 ; Rebillard, 2007 ; Smyrnaios, Marty, 2017).

Du point de vue des sujets, un fort déséquilibre se remarque également entre les articles les plus commentés et les autres. Ainsi 59 sujets ne comportent qu'entre 1 et 5 commentaires, 34 entre 5 et 10 et 14 sujets seulement sont commentés plus de 10 fois. Là encore nous reviendrons sur les raisons pouvant expliquer ces différences, mais nous pouvons d'ores et déjà noter que les sujets les plus commentés sont ceux qui concentrent le plus de commentateurs occasionnels et très occasionnels pour les pages consacrées à la commune.

Pour procéder à l'analyse de ces chiffres nous avons étudié les commentaires en essayant de comprendre les positionnements des commentateurs et en utilisant le cadrage développé plus haut. Plusieurs résultats nous semblent devoir être soulignés. Tout d'abord il convient de noter que les commentateurs les plus assidus sont clairement impliqués dans le débat municipal. Ainsi, parmi les 11 principaux commentateurs, 10 d'entre eux peuvent être clairement identifiés comme des soutiens à des listes, voire comme partie prenante à l'élection ${ }^{5}$. Cinq d'entre eux ont accepté de nous rencontrer pour évoquer leurs pratiques de commentateurs durant l'élection. Ils ont été d'une façon ou d'une autre partie prenante de la campagne (présence sur une liste, distribution de tracts, conjoint sur une liste, membre d'un parti impliqué dans la campagne). Il est vrai que lors de cette élection municipale ce ne sont pas moins de 132 candidats qui étaient présents sur les 4 listes. Seul Jean Mouclade, parmi les commentateurs à plus de 10 commentaires, ne peut pas être caractérisé par ses prises de position dans la campagne municipale ${ }^{6}$.

Deux commentateurs à plus de 40 posts se réclament ouvertement de l'UMP et l'un d'entre eux était d'ailleurs candidat sur la liste d'union de la droite. Le PRG pouvait compter sur deux commentateurs, l'un à 18 posts et l'autre à 12 . Les soutiens du FN étaient au nombre de trois, dont la tête de liste réagissant sans pseudonyme (22, 18 et 10 posts). Enfin le maire socialiste sortant avait pour soutien trois commentateurs, dont le principal contributeur, 81 posts sur la période. Les 3 principaux commentateurs, en dehors du candidat FN, font partie de ceux que nous avons identifiés et nous pouvons remarquer qu'ils ne s'expriment par ailleurs pas dans l'espace public $\operatorname{local}^{7}$, même s'ils sont régulièrement présents dans les réunions politiques.

Nous nous sommes également aperçu que le type d'article, selon la classification présentée plus haut, n'était pas déterminant quant à la teneur des commentaires, à l'exception de ceux traitant de faits divers. L'identité des commentateurs est en revanche bien plus importante car il s'avère que leurs contributions successives s'organisent quasi systématiquement autour d'une même thématique. Deux communautés distinctes sont présentes et ne se rencontrent que rarement. La première est caractérisée par sa connaissance affichée du contexte local et surtout des candidats à l'élection municipale ; la seconde n'intervient que sur un seul type de sujet et propose une interprétation nationale des évènements locaux. Les contributions s'organisent ainsi autour de trois grandes thématiques :

- la volonté de compléter l'information, voire de révéler des faits que l'on ne trouve pas dans les colonnes de La Dépêche du Midi, mais toujours avec un objectif partisan.

- l'opposition à la ligne éditoriale du journal, en particulier à son parti-pris politique.

- la réaction aux faits-divers.

Avant de commencer l'analyse des commentaires il faut d'abord revenir sur les pratiques de modération du journal qui vont au-delà de ce qui est affiché comme constituant les règles de participation.

Ainsi, durant la campagne des municipales, de nombreux commentaires respectant la charte mais au contenu politique en contradiction avec la ligne du journal seront effacés. Nous avons en effet effectué une sauvegarde plusieurs fois par jour des articles et des commentaires et nous avons comptabilisé 57 commentaires supprimés, la plupart respectant en tous points la charte ${ }^{8}$. Il s'agit toujours de commentaires remettant en question la politique du Conseil Général, l'action et le comportement des candidats radicaux aux élections municipales ou la ligne éditoriale partisane de La Dépêche. Ce commentaire du 31/01/2014 est par exemple posté à 8 h43 et a disparu a 10 h57 : «P. Guillamat est satisfait, il enregistre 
qu'il va y avoir UNE embauche à Moissac. Par contre sous la rubrique de Lizac, il y a l'annonce d'un spectacle qui a lieu ce soir au Hall de Paris : c'est un spectacle de la ville de Moissac, mais il est vrai que la culture fait peur au poulain de la DDM et qu'il ne faut pas trop en parler " sur «Les conseillers généraux visitent les ateliers de Gillis Aéro en compagnie de Serge Dumas ». La modération pour des raisons de politique locale est donc une pratique constatée par nos observations mais qu'aucun acteur du journal n'a souhaité expliquer. Les commentateurs s'en sont souvent émus "Un de mes deux commentaires a disparu. On se croit en URSS. Pas droit à la parole si on émet des doutes réels sur cette équipe. Bravo cet esprit démocratique " unjeunequivoteamoissac, 23/02/2014, 8h09, sur « Pierre Guillamat, une seule liste d'avenir, la nôtre » (22/02/2014).

Cette pratique doit bien sûr être prise en compte pour comprendre la réaction des commentateurs les plus assidus qui interviennent sur la situation locale car ils en sont les principales victimes.

\section{CORRIGER ET COMPLÉTER LE JOURNAL}

Le premier enseignement à tirer de l'examen de l'ensemble des commentaires est la volonté de transmettre des informations que les commentateurs assidus estiment ne pas être traitées par le journal. Ainsi, ceux qui sont visiblement insérés dans le contexte local, utilisent l'espace qui leur est offert par La Dépêche du Midi pour communiquer des éléments concernant la commune et particulièrement sa vie politique et ses enjeux. Deux thématiques principales ont été repérées. Il s'agit d'abord de l'évocation du bilan des mandatures municipales précédentes, qui peut être faite de façon positive, mais qui le plus souvent insiste sur des points négatifs pas forcément mis en avant par La Dépêche du Midi (de nombreux posts émanant des soutiens de la liste UMP insistent par exemple sur la dette de la ville ${ }^{9}$ ) :

"le déficit est maintenant de près de 12 à 15 millions d'euros : il n'était pas responsable du budget grand corps malade " Noelle de Moissac, 16/01/2014, 12h51 sur «Pierre Guillamat : La campagne se poursuit ».16/01/2104

Il s'agit dans la plupart des cas d'opinions même si les chiffres mentionnés dans le post précédent sont exacts et ne sont jamais mentionnés dans les colonnes du journal. Ils sont en revanche présents sur les documents de propagande de la liste UMP.

On trouve ensuite plusieurs comptes rendus de la campagne en train de se dérouler. Ces derniers présentent des points qui ne sont pas, ou pas assez précisément, présentés par les journalistes aux yeux des commentateurs engagés. Il s'agit par exemple de descriptions de réunions publiques ou bien d'épisodes de campagne passés sous silence par le journal.

« je suis allé ce soir à la réunion de la droite de Moissac au Centre Culturel. Grande surprise : j’ai compté 151 personnes desquelles il fallait enlever 33 colistiers. 118 personnes dont je fais partie et qui ont assistés à une vraie info, dynamique et faite par des intervenants compétents... " (unjeunequivoteamoissac, 28/02/2014 14h26, sur « Le chasselas reste le meilleur ambassadeur de la ville ", $28 / 02 / 2014$

Le principal élément factuel et vérifié ayant trait à cette campagne, et que ni La Dépêche du Midi ni les différents documents de propagande n’ont évoqué, est l'accident vasculaire cérébral dont a été victime le candidat du PRG au début du mois de janvier. Cet événement qui l'a tenu éloigné de la ville durant plus d'un mois a ainsi été largement mis en avant par les commentateurs qui ont participé à publiciser ce qui était, à ce moment-là ${ }^{10}$, nié par l'entourage du candidat.

«Suivant mes sources, G. a fait un AVC en janvier, suivi d'une hospitalisation. Pour l'avoir vu il y a 15 jours, il est fatigué et ne tient pas la longueur, ce qui est normal après un AVC. Je vais voter et je veux savoir si votre candidat est encore en état physique " (JD82200, 04/03/2014, 19h57 sur « La créance du Lutosa toujours en question » 04/03/2014)

"Belle photo du revenant mourant " Noelle de Moissac, 19/01/2014 9h01 sur « Dernier vœux de la mandature », 19/01/2014

Les commentaires de ces internautes témoignent donc d'abord d'une intention de dévoilement à l'égard d'informations qui sont volontairement cachées par le quotidien régional mais aussi d'une visée de contre-propagande à l'égard d'un journal jugé trop partisan. Beaucoup de commentateurs remettent ainsi en cause la ligne éditoriale du journal et tentent de "rectifier son discours " pour reprendre le constat de Laura Calabrese (2014).

\section{L'OPPOSITION À LA LIGNE ÉDITORIALE : LE RADICALISME EN QUESTION}

Il apparaît en effet que, si l'on comptabilise les thèmes traités par les commentateurs les plus assidus localement, on s'aperçoit rapidement que la principale 
raison de contribuer réside dans l'opposition à la ligne éditoriale du journal. Ainsi, sous 55 articles sur 93, on repère des commentaires critiquant ouvertement $\mathrm{La}$ Dépêche du Midi et sa façon de traiter l'actualité locale. De même, on retrouve 50 articles sous lesquels ont été postés des commentaires remettant en cause le positionnement politique du PRG, ses candidats ou l'action du président du Conseil Général de Tarn et Garonne par ailleurs PDG du journal. Cela se fait le plus souvent par des moyens détournés ou grâce à l'obstination ${ }^{11}$ pour éviter de voir son texte disparaître ou ne pas être publié.

« et pourquoi le prendre lui en photo ? il n'est pas maire, il n'est pas adjoint, il est juste simple conseiller : ah oui, j’oubliais, La Pravda » (jd82200, 19/01/2014 18h16 sur « Derniers vœux de la mandature »).

\section{«A Moissac parce que le candidat de la DDM a suspendu ses activités électoralistes, suite à un coup de pompe où la pompe en a pris un coup, la conséquence directe est la disparition de toute autre information sur la ville, sauf le sport ou à la rigueur la sécurité. C’est une conception de la déontologie journalistique qui n’a d'égale que la sérénité des radicaux à œuvrer dans un cadre de saine vérité "Oza- guay, 13/01/2014 13h09 sur « JL Jun transmet toujours sa passion du Judo », 12/01/2014.}

La permanence d'un courant radical dans le Tarn-et-Garonne et singulièrement dans la seconde circonscription législative de ce département est une singularité politique que nous ne pouvons ni analyser ni remettre en perspective dans le cadre de cet article mais qui exerce une influence majeure sur la situation politique locale et par conséquent sur les positionnements que nous pouvons observer. La Dépêche du Midi étant liée organiquement par la personne de son PDG à ce mouvement, ce contexte structure les interventions de beaucoup d'internautes au courant du contexte local.

Ces deux premiers thèmes s'inscrivent parfaitement dans les analyses faites par ailleurs sur le méta-discours produit par les internautes qui corrigent, critiquent, complètent et prennent de la distance avec la production journalistique (Calabrese, 2016).

Les références à la déontologie journalistique, mais aussi au journal soviétique Pravda sont fréquentes et partagées par presque tous les commentateurs assidus. Il convient d'ailleurs de noter que malgré leur soutien à des listes différentes pour cette élection municipale, l'ensemble de ces commentateurs (à l'exception de ceux qui soutiennent les candidats radicaux) se retrouvent fréquemment sur des positions similaires dans la critique du journal. Il est même notable que les quelques échanges qu'ils entament les uns avec les autres s'achèvent tous sur une sorte de consensus pour critiquer la ligne éditoriale du journal.

Notons enfin qu'un dialogue existe entre ces 11 commentateurs (y compris les 2 soutiens du PRG) et que, en dépit de quelques invectives, il se déroule avec des efforts d'argumentation. Ces efforts argumentatifs semblent cependant plus destinés à convaincre les éventuels lecteurs que les autres commentateurs.

Néanmoins, le nombre très réduit de participants ne nous permet pas de parler de public. En outre, la discussion, même plutôt cordiale et argumentée a lieu entre des personnes engagées et soutenant une liste. Aucun ne change d'avis. Ils participent d'abord avec pour objectif de convaincre des électeurs et de dénoncer le discours partisan du journal. Ils partagent tous, hormis les 2 commentateurs radicaux, la même position sur la ligne éditoriale du journal et déplorent l’hégémonie politique du PRG sur le département.

Il est cependant nouveau, et ce n'est pas l'un des moindres apports de la section commentaires, d'apercevoir une polyphonie énonciative politique sous la bannière de La Dépêche du Midi, même en ligne, parfois censurée et dans un espace subalterne.

\section{LA POLITISATION PAR LE COMMENTAIRE DES FAITS DIVERS}

La grande majorité des commentateurs (tous ceux ayant posté moins de 5 fois sur la période dans les pages Moissac) n'ont pris aucune position sur la campagne municipale en tant que telle. Ils ont en revanche tous réagi sur un même type de sujet. Ainsi on retrouve les posts de la quasi-totalité de ces commentateurs sous des faits divers relatant des vols et des agressions, le plus souvent pour les déplorer et pour insister sur leur impression de dégradation de la sécurité sur le territoire.

L'ensemble de ces contributeurs se mobilisant sur les faits divers est constitué de commentateurs que nous avons classés comme occasionnels ${ }^{12}$ mais la plupart sont très impliqués sur l'ensemble du site (Monterosso : 1174 commentaires ; Homosapiens : 8755 commentaires ; LGR : 1868 commentaires ; Agnostique : 1063 commentaires ; Aergselrahc : 1788 commentaires ; Roberto : 1217 commentaires ; Xavi31 : 3045 commentaires). Il est impossible de tirer des conclusions trop générales mais il semble qu'il existe un ensemble de commentateurs spéciali- 
sés dans les faits divers, qui interviennent quel que soit le territoire concerné.

Un article sur un fait divers relatant une altercation entre un présumé voleur et une personne ayant pris l'initiative de le suivre dans la rue alors qu'aucun acte délictueux n'avait été commis a ainsi recueilli 51 commentaires de 42 contributeurs différents, tous ou presque défendant l'apprenti détective ${ }^{13}$ :

"Dès qu’il se passe un problème de ce genre où que se soit il ne faut rien faire et baisser les bras de peur de donner de la vivacité au FN. Pour le moment la justice en France est pourrie et inefficace et si ca continue les gens s'organiseront et créeront leur propre milice". apollo14, le 25/01/2014

\section{« L'agresseur relâché le lendemain}

\section{UNE HONTE ! ON PEUT IMAGINER LA PEUR DES VICTIMES !!! » peggysue, le 23/01/2014}

"Il serait enfin temps de réagir après des mois statiques......!!!!!!!!!»

Homosapiens (8755 commentaires)
le $22 / 01 / 2014$

"Ca suffit en centre fermé ! Et comme cadeau pour les 18 ans : au trou!»

Agnostique (1063 commentaires)

le $22 / 01 / 2014$

« ceci n'est pas de la pub pour le FN mais juste une réalité de la vie de tout les jours pour certaines personnes mais que certains bobos ont du mal à admettre, pourtant les chiffres sont la et personne ne fait rien...pauvre France " purvillefranchois, le 22/01/2014

"Voilà un exemple clair de la justice en France avec ces jeunes voleurs violents identifiés et libres. Comment voulez vous vous sentir en sécurité ?

Comment voulez vous faire confiance a la police et a la justice ? La situation est grave et je finis par comprendre les personnes qui n'interviennent pas lors de violence. L'état est fortement coupable. " xavi31 (3473 commentaires)

le $22 / 01 / 2014$

« c'est regretable de voir que l'agresseur soit deja en liberte et qu'il risque de se venger " wistiti1200 (5843 commentaires)

le $22 / 01 / 2014$
De même, sur les 10 autres articles ayant recueilli plus de 10 commentaires, 5 sont des faits divers relatant, qui un vol de pelle mécanique, qui la dégradation du bras d'une statue de vierge, ou qui l'interpellation d'un conducteur ivre...

Plusieurs traits intéressants sont présents dans les exemples sélectionnés ci-dessus et sont significatifs quant à la façon dont ces commentaires sont construits :

- ils s'inscrivent pour la plupart d'entre eux dans le registre de l'émotion. Que ce soit la compassion, la peur, l'indignation ou la colère, les émotions sont systématiquement convoquées. L'utilisation des points d'exclamation, des phrases courtes et des majuscules en est une illustration.

- ils sont également rattachables au registre de la spontanéité. Il ne s'agit pas de posts argumentés ou appelant une réponse. Ils sont présentés comme une réaction spontanée.

Ces deux caractéristiques correspondent à celles décrites par Michael Schudson lorsqu’il évoque le modèle de conversation fondé sur la sociabilité, qu’il oppose à celui s'attachant à la résolution de problèmes (1997). Ce modèle repose sur l'expression publique et le partage d'opinions par l'intermédiaire de la mise en avant d'émotions.

Ces commentaires ne donnent en outre aucune information, ni ne remettent en question la ligne éditoriale du journal. Ils ne prennent pas non plus partie dans la campagne électorale. S'ils citent un parti politique, le Front National, c'est pour ne pas s'en réclamer ouvertement (« ceci n'est pas une pub pour le $\mathrm{FN} »$ ) ou pour souligner la peur qu'il inspirerait aux pouvoirs publics (« baisser les bras de peur de donner de la vivacité au FN »). On comprend bien cependant que ces commentateurs ont conscience de développer les thèmes qui sont ceux du Front National, mais ils refusent d'en porter l'étendard. On retrouve là l'un des traits caractéristiques des discours sur "les bonnes questions " que poserait ce parti d'extrême droite, que l'on peut analyser comme une légitimation par la bande.

Beaucoup de ces commentateurs qui interviennent sur les faits divers pour déplorer l'insécurité sous-entendent en outre, plus ou moins directement, que ces phénomènes doivent être liés à la présence de présumés étrangers :

« si on en sulfate un, il y a marche blanche, alors il faudrait qu'il $y$ ait beaucoup de marches blanches » bookerjjn, 22/01/2014, $8 \mathrm{~h} 47$ sur « Surpris en plein cambriolage, ils 
brisent les vitres de la voiture du témoin " $22 / 01 / 2014$

« moissac, même dans l'aveyron on connaît. C'est bien là où se trouve le marché du chameau et des babouches " purvillefranchois, 29/01/2014 13 h10 sur « Un agent recenseur se fait voler sa sacoche », 29/01/2014.

« comme ca ils pourront continuer leurs méfaits et doivent bien rigoler entre eux »nordique, 24/01/2014 10h28 sur « L'agresseur de la cité du Maroc ira en justice » ${ }^{14}, 22 / 04 / 2014$.

«Pauvres gens, ne croyés pas que cela va s’arranger, malheureusement avec les nouvelles lois de notre ministre de la justice (vous la connaissez j'espère)il en faudra des grosses bétises pour qu'ils soient bien sanctionnés, et sa eux ils l'ont bien entendu ». Tarzan47, sur «Surpris en plein cambriolage, ils brisent les vitres de la voiture du témoin » le 22/01/2014

La première remarque relative à cette série de posts concerne le caractère identitaire des pseudonymes : " purvillefranchois " se référant assez explicitement à une origine exempte de tout métissage, « Nordique » se définissant en opposition aux populations venant du Sud et " Tarzan47 » associant un département avec un héros blanc évoluant dans un environnement hostile car non civilisé.

L’assimilation de la délinquance aux populations étrangères est totalement assumée et n'est même presque plus du registre de l'implicite même si elle fonctionne toujours en utilisant le registre de l'allusion. Il est intéressant de noter que sur aucun des articles commentés il n'est fait mention des délinquants, ni par leur origine, ni par leur nom. L'assignation identitaire se fait pourtant quasi automatiquement dans la section commentaire. Celui de « purvillefranchois » sous un article mentionnant un vol dont le journaliste ne sait rien à part qu'il a eu lieu est à ce tire exemplaire. Il lui suffit de caractériser la ville par une référence fantasmée à la présence d'un marché identique à ceux de l'Afrique du Nord pour que ce fait divers prenne une caractéristique nouvelle : "là où se trouve le marché du chameau et des babouches ». Selon François Rastier, qui propose des critères d'identification des sites racistes, "l'allusion », comme "l'euphémisation témoigne », à l'intérieur de ces sites «que nous sommes sous le régime de la censure mais que nous pouvons la contourner» (2006, p.82).

Ces commentaires activent aussi un clivage entre " eux" et "nous » : "si on en sulfate UN", «ILS pourront continuer leurs méfaits ", " et sa (sic !) $E U X$, ils le savent bien ». Jamais il n'est fait une référence directe à l'identité ethnique, cependant l'ensemble du paratexte et du contexte amène le lecteur à comprendre à qui «ils ", " eux " et «un » font référence. D’abord ils sont différents du rédacteur du message qui, à travers son pseudonyme, se décrit lui-même avec des références identitaires, ensuite ils sont lus dans un ensemble de commentaires tels ceux de "Purvillefranchois" beaucoup plus explicites sur le sujet, et enfin ils s'inscrivent dans un espace public national où les succès électoraux du FN sont très présents et où les discours d'assignation d'identité en fonction des origines ethniques réelles ou supposées sont devenus courants. Cette opposition entre " eux " et «nous » est, elle, aussi caractéristique des discours racistes (Rastier, 2006).

Plus étonnant, car a priori interdit par la charte de modération, mais aussi par la loi, l'appel détourné à la violence est présent dans certains commentaires. Celui de «Bookerjjn "sur «les marches blanches ", dont il déplore la rareté, démontre parfaitement que certains commentateurs parviennent à contourner la barrière de la modération. Nous n'avons malheureusement pas pu avoir accès aux messages modérés a priori, qui, même s'ils ne rentraient pas dans notre corpus, auraient pu nous en apprendre davantage sur l'importance quantitative de ce type de message, sur leurs auteurs et sur les éventuelles stratégies de contournement dont pourraient témoigner les messages publiés.

Centrées sur l'insécurité et la dénonciation d'une présence étrangère trop nombreuse, ces contributions épousent les thématiques popularisées par l'extrême droite française depuis plusieurs décennies. Deux types d'hypothèses, plus complémentaires qu'opposées, peuvent être avancées pour analyser cette situation.

La première concerne la ligne éditoriale de la PQR dont on sait qu'elle fait la part belle aux fait-divers (Berthaut, Darras, Laurens, 2009). La construction idéologique de l'extrême droite reposant en partie sur l'exploitation et la circulation de faits-divers tendant à démontrer par l'exemple la situation d'insécurité en France et le laxisme des autorités, celle-ci la rencontre et s'en nourrit sans difficulté.

L'extrême prolixité de certains commentateurs, qui se spécialisent en faits-divers, leur façon de ne jamais endosser des habits partisans mais simplement de s'indigner et de compatir, nous engage à nous demander s'il ne serait pas possible de leur assigner une identité partisane et d'interpréter ces commentaires comme le résultat d'une stratégie. Si tel était le cas nous serions donc face à une offensive parti- 
culièrement habile de certains individus, politisant volontairement le local par l'intermédiaire de faits quotidiens. Cette expression est cependant beaucoup plus policée que ce que la recherche contemporaine a identifié comme étant celle des militants d'extrême droite quand ils s'expriment librement (Bo, 2015 ; Hobeika, Villeneuve, 2017). En outre, il s'agit ici clairement d'une utilisation de l'information médiatique et non d'une pratique de ré-information telle que la met en œuvre l'extrême droite contemporaine (Voirol, 2017).

L’accumulation des faits-divers accompagnée des interprétations des commentateurs propose ainsi une démonstration politique des thèses de l'extrême droite, sur un média classique et débarrassée des excès de langage habituellement constatés.

\section{Conclusion}

Il nous semble que ces résultats permettent d'apporter quelques éclairages à notre questionnement sur la constitution de publics locaux et sur la politisation de la vie locale. Deux groupes de commentateurs, entretenant entre eux des rapports bien distincts sont visibles dans la section commentaires de La Dépêche du Midi pour la commune de Moissac.

Le premier est constitué par un ensemble de commentateurs qui partagent deux caractéristiques : une bonne connaissance de la vie politique locale, voire une implication dans son fonctionnement pour certains d'entre eux, et une capacité à se reconnaître dans le jeu politique partisan national. Ainsi ces lecteurs du journal en ligne échangent sur la campagne municipale en nourrissant leurs commentaires de leurs connaissances du terrain et de leurs observations. Ils interviennent quasi quotidiennement et sur tous les articles publiés, quel que soit le sujet.

Ils sont ainsi en capacité d'apporter des compléments au traitement de la campagne de La Dépêche et ils remettent fréquemment en question sa ligne éditoriale. Il est cependant à noter que le type de commentaire qu'ils postent sur la campagne municipale personnalise fortement l'élection. Ils construisent par conséquent un cadre interprétatif de l'élection qui se rapproche de celui que la science politique qualifie de " course de chevaux » en faisant référence au rôle des sondages et de leur utilisation par les médias (Lehingue, 2007). Pour eux il s'agit bien d'une lutte entre 4 personnalités. De ce point de vue-là, ils sont donc en accord avec les cadrages médiatiques dominants (Marty, 2015) même s'ils s'insurgent contre le traitement de la campagne par le journal.
Ces individus sont ceux qui fréquentent les réunions publiques, le monde associatif, les partis politiques. Ils sont insérés dans la vie locale et pourraient être qualifiés d'autochtones selon la terminologie de la science politique (Retiere, 2003 ; Mischi, Renahy, 2008). Ils représentent un tout petit groupe de commentateurs (entre 10 et 15) qui sont en capacité d'articuler les niveaux locaux et nationaux en terme de politique partisane. Chacun défend une liste et est capable d'argumenter une montée en généralité à partir de la situation locale. Il n'est pas du tout inattendu de retrouver ce type de personnes, idéal-typique par rapport aux représentations que les journalistes eux-mêmes se font de leurs lecteurs (Bousquet, Marty, Smyrnaios, 2015). Ce groupe de commentateurs est cependant très réduit et utilise principalement la section commentaires comme une tribune. Il ne rencontre donc que difficilement la notion de public. Il s'oppose nettement au second groupe, caractérisé par une relation plus médiatisée et lointaine avec le contexte politique local.

Ainsi le second groupe que l'on a observé est moins attendu. Tout d'abord il est beaucoup plus nombreux, constitué pour les pages que nous avons étudiées par une centaine de personnes ${ }^{15}$. Ensuite il n'intervient que sur certains sujets très précis, les faits divers. Il ne témoigne pas d'une connaissance fine du contexte local, voire, dans la plupart des cas, il est possible de déceler une absence complète de savoir sur la situation politique, sociale ou économique de la commune. Il ne connaît pas non plus les candidats locaux à l'élection. Aucun d'entre eux n'est cité dans les posts de ces commentateurs. Le registre des interventions est plus émotionnel, il ne recherche pas l'argumentation et se contente de la compassion envers les victimes de vols ou de violence et de l'indignation vis-à-vis de ceux qui commettent des actes délictueux. Ce groupe s'inscrit pleinement dans une vision de l'actualité et de son commentaire marqué par le pathos et la vie quotidienne. En apparence, ces commentateurs qui se répondent fréquemment, semblent ne partager que leurs émotions et une vision de la réalité très " terre à terre ". Pourtant eux aussi opèrent à une montée en généralité, moins, voire non revendiquée, et à une articulation entre des faits locaux et une situation nationale qui permet une politisation forte du local. Cette politisation rencontre en France les thématiques de l'insécurité et de l'inquiétude identitaire, qui sont le fonds de commerce du Front National dans le débat politique national mais infusent de plus en plus dans le discours d'autres partis. Le public observé dans ces commentaires n'est pas caractérisable territorialement mais il se retrouve sur le local, se nourrit du local, avec les implications en termes de proximité (Le Bart, Lefevbre, 2005) et de quotidienneté que cela comporte. Proche à pre- 
mière vue du «Public fantôme " de Lippmann, éloigné de la complexité politique, ce groupe constitue pourtant une communauté partageant une préoccupation centrale qu'il exprime de façon récurrente et construit dans l'interaction. Ainsi à travers ses commentaires sur les faits divers, ce groupe prend position et propose une clé politique de compréhension d'un monde complexe.

Nous sommes donc en face de groupes de commentateurs, tous deux capables d'opérer une politisation de l'actualité de cette commune mais selon deux logiques très différentes. La première correspond aux canons de l'interprétation de la vie politique locale en terme d'autochtonie, que la recherche contemporaine décrit comme en voie de disparition (Retiere, 2003) et la seconde, que nous ne nous attendions pas à rencontrer, est capable d'ancrer la vie locale dans une vision du monde nationale voire internationale avec des embrayeurs de sens (Georges, 2009) construits autour des faits divers et de la vie quotidienne.

Ce résultat doit être mis en perspective avec ce qu'écrit G. Carbou à propos du rôle important que peuvent jouer les espaces de commentaires pour la constitution de publics de commentateurs mais aussi et surtout de lecteurs :

" Nous avons ainsi plutôt affaire à des arènes discursives où les points de vue se construisent, se renforcent, et se perpétuent. Le simple lecteur des commentaires $y$ affermit - ou y découvre - une vision du monde consistante et le contributeur y éprouve la satisfaction de l'intersubjectivité. " (2015, p. 196).

Cette politisation du local mériterait par conséquent d'être approfondie par une étude systématique des commentaires de l'intégralité des pages locales de ce site et de ceux d'autres représentants de ce type de presse car elle pourrait jouer un rôle important dans la constitution des enjeux politiques locaux.

Date de soumission de l'article : 30 mars 2017 Date d'acceptation : 14 juin 2018

\section{NoTES}

${ }^{1}$ A partir du moment où la taille du territoire concerné implique qu'il ne peut y avoir de relations interpersonnellles et de rencontre entre tous ceux qui en font partie, les médias sont l'un des acteurs majeurs de la formation des publics (Dewey, 1927). ${ }^{2}$ D'abord directrice de cabinet du Conseil Général de Tarn et Garonne, puis député de la seconde circonscription de ce département, Sylvia Pinel est devenue ministre des gouvernements Ayrault et Valls et a également succédé à J-M Baylet comme présidente du Parti Radical de Gauche.

${ }^{3}$ Un document interne, édictant et rentrant dans les détails des règles de la modération que la société Atchik s'engage à appliquer, lie les deux partis, mais il nous a été impossible d'y accéder.

${ }^{4}$ Il faut noter qu'il n'y a aucune interaction avec les journalistes, ceux-ci n'intervenant jamais dans les commentaires malgré plusieurs interpellations.

${ }^{5}$ Notre connaissance du contexte local et de ses acteurs nous a permis d'identifier plusieurs commentateurs. 6 ont confirmés qu'ils étaient bien les auteurs des commentaires et 5 ont accepté de nous rencontrer, en juillet 2016, soit plus de 2 ans après l'élection. La plupart (3 sur 6$)$ ont par ailleurs cessé de commenter et les autres ne le font qu'à une fréquence beaucoup plus espacée.

${ }^{6}$ Il faut savoir qu'une quinzaine de commentateurs empruntant le nom de "Mouclade" mais avec des prénoms divers participent sur l'ensemble du site de La Dépêche du Midi en intervenant tous azimuts et de façon délibérément humoristique. Leur objectif est visiblement celui de se moquer à la fois des articles et des commentaires. Un exemple, sous la photo de l'ensemble de la liste du candidat radical présentée dans La Dépêche du Midi, le commentaire de Jean Mouclade était : "Pas une seule quenelle sur la photo. Bravo. " (commentaire du 22/02/2014). Ainsi le commentateur « Jean Mouclade » intervient généra- lement sur les articles concernant le Tarn-et-Garonne alors que d'autres «Mouclade» sont actifs sur d'autres territoires. Il est parfois pris à parti par les autres commentateurs qui lui reprochent son manque de sérieux, mais il intervient régulièrement sur tous les sujets les plus commentés.

${ }^{7}$ Par l'intermédiaire des médias, dans des réunions publiques ou sur les réseaux socio-numériques.

${ }^{8}$ Cela représente pourtant déjà plus de $10 \%$ du total des commentaires publiés.

${ }^{9}$ Il convient de noter que ce sujet n'est pas évoqué par le candidat radical car il était l'adjoint aux finances du maire socialiste jusqu'à la fin de l'année 2013. La Dépêche du Midi évite donc aussi ce sujet.

${ }^{10}$ Le candidat lui-même a reconnu cet AVC après la fin de la campagne.

${ }^{11}$ Ainsi, un même commentaire de NoelledeMoissac a été posté plus de 12 fois, il a 11 fois été supprimé mais est resté sur le site la 12ème fois, utilisant une tactique bien décrite par Smyrnaios et Marty dans leur article sur les « nettoyeurs du net »(2017).

${ }^{12}$ Car ils n'interviennent qu'une fois ou deux dans la rubrique de cette commune.

${ }^{13}$ « Surpris en plein cambriolage, ils brisent les vitres de la voiture du témoin », 22 janvier 2014.

${ }^{14}$ La « Cité du Maroc » est un quartier de la ville nommé ainsi car construit après un don du roi du Maroc faisant suite à des inondations destructrices en 1930. Rien à voir donc avec l'origine de l'agresseur présumé.

${ }^{15}$ Chiffre qu'il convient cependant de relativiser car ces commentateurs interviennent sur l'ensemble du site et non sur les seules pages d'une ville comme les précédents. 


\section{RÉFÉRENCES BIBLIOGRAPHIQUES}

Agulhon, M., 1979 [1970], La République au village. Les Populations du Var de la Révolution à la Seconde République, Paris, Le Seuil.

Amiel, P., 2017, « Le journalisme de solutions », Communication [En ligne], vol. 34/2, consulté le 20 oct. 2017, http:// communication.revues.org/7226.

Aubert, A., 2011, « Le participatif perçu par les professionnels du journalisme : état des lieux », Les Cahiers du journalisme, no 22-23, pp. 42-55.

Ballarini, L., 2008, " Presse locale : un média de diversion ", Réseaux, no 148-149, pp. 405-426.

Bergström, A., Wadbgring, I., 2014, « Beneficial Yet Crappy: Journalists and Audiences on Obstacles and Opportunities in Reader Comments », European Journal of Communication.

Berthaut, J., Darras, E., Laurens, S., 2009, « Pourquoi les faits divers stigmatisent-ils ? L’hypothèse de la discrimination indirecte », Réseaux, no 157-158, pp. 89-124.

Boczkowski, P., Mitchelstein, E., 2012, « How Users Take Advantage of Different Forms of Interactivity on Online News Sites : Clicking, E-Mailing, and Commenting ", Human Communication Research, vol. 38, no 1, pp. 1-22.

Bousquet, F, Marty, E., Smyrnaios, N., 2015, « Les nouveaux acteurs en ligne de l'information locale : vers une relation aux publics renouvelée ? ", Sur le journalisme About Journalism Sobre jornalismo, vol. 4, no 2, pp. 48-61.

Boyadjian, J., 2015, « Les usages frontistes du Web », in Crépon, S., Dézé, A., Mayer, N. (Éds.), Les faux-semblants du Front National, Paris, Presses de Sciences Po (P.F.N.S.P), pp. 141-160.

Calabrese, L., 2016, " "Réfléchissez avant d'écrire !”, approximation et précision dans le discours des lecteurs de la presse en ligne ", Shyldkrot, H. B.-Z., Adler, S., Asnes, M., Nouveaux regards sur l'approximation, Honoré Champion, Paris.

Calabrese, L., 2014, " Rectifier le discours d'information médiatique. Quelle légitimité pour le discours profane dans la presse d'information en ligne ? ", Les Carnets du Cediscor, no 12, pp. 21-34.

Carbou, G., 2015, « Presse en ligne et communautés cognitives : analyse des sections commentaires d'Atlantico.fr et de l’Humanité.fr », Réseaux, no 195/5, pp. 194-223.

Charaudeau, P., Maingueneau, D. (Éds.), 2002, Dictionnaire d'analyse du discours, Paris, Seuil.

De La Haye, Y., 1984, Dissonances, critique de la communication, La pensée sauvage.

Dewey, J., 2010 [1927], Le Public et ses problèmes, Paris, Gallimard Folio.

Dupret, B., Klauss, E., Ghazzal, Z., 2010, « Commenter l'actualité sur Internet », Réseaux, no 160-161, pp. 285317.

Estienne, Y., 2007, Le Journalisme après Internet, Paris, L'Harmattan.

Frisque, C., 2010, «Une reconfiguration des espaces médiatiques et politiques locaux ", Revue française de science politique, vol. 60, no 5, pp. 951-973.
Georges, F., 2009, « Représentation de soi et identité numérique : Une approche sémiotique et quantitative de l'emprise culturelle du Web 2.0 ", Réseaux, no 154, La Découverte, pp. 165-193.

Graham, T., Wright, S., 2015, " A Tale of Two Stories from "Below the Line" : Comment Fields at The Guardian », The International Journal of Press/Politics, no 20, pp. 317-338.

Hamidi, C., 2006, « Éléments pour une approche interactionniste de la politisation. Engagement associatif et rapport au politique dans des associations locales issues de l'immigration », Revue française de science politique, vol. 56, no 1, pp. 5-25.

Hermida, A., Thurman, N., 2008, " A Clash of Cultures. The Integration of Usergenerated Content within Professional Journalistic Frameworks at British Newspaper Websites », Journalism Practice, vol. 2, n 3, pp. 343-356.

Hermida, A., Domingo, D., Heinoven, A., Paulussen, S., Quandt, T., Reich, Z., Singer, J., Vujnovic, M., 2011, «The Active Recipient : Participatory Journalism Through the Lens of the Dewey-Lippmann Debate », ISOJ.

Hobeika, A., Villeneuve, G., 2017, « Une communication par les marges du parti ? », Réseaux, no 202-203, pp. 213240 .

Jouët, J., Le Carroff, C., 2013, « L’actualité politique et la participation en ligne ", in Jouët, J., Rieffel, R. (Éds.), S'informer à l'ère numérique, Rennes, PUR, pp. 117-157.

Le Bart, C., Lefebvre, R., (Éds.), 2005, La proximité en politique, Rennes, PUR.

Le Bohec, J., 2000, « La Question du "rôle démocratique" de la presse locale en France ", Hermès, no 26-27, pp. 185-198.

Lehingue, P., 2007, "L'emprise des sondages sur le jeu électoral, vrais et faux débats », Savoir Agir, no 1, pp. 37 47.

Lippmann, W., 2008 [1925], Le public fantôme, Demopolis.

Marty, E., 2015, « Les élections municipales au miroir de la presse quotidienne régionale. Des cadres médiatiques aux thématiques politiques ", Mots. Les langages du politique, no 108, pp. 39-55.

Miège, B., 1990, La Société conquise par la Communication, Grenoble, PUG.

Mischi, J., Renahy, N., 2008, "Pour une sociologie politique des mondes ruraux », Politix, no 83, pp. 9-21.

Norris, P., 2001, Digital Divide: Civic Engagement, Information Poverty, and the Internet Worldwide, New York, Cambridge University Press.

Pierru, E., Vignon, S., 2008, « L'inconnue de l'équation FN ; ruralité et extrême droite. Quelques éléments sur le département de la Somme », in Antoine, A., Mischi, J. (Éds.), Sociabilité et politique en milieu rural, Rennes, PUR.

Rastier, F., 2006, "Sémiotique des sites racistes ", Mots, no 80, pp. 73-85.

Rebillard, F., 2007, Le web 2.0 en perspective : une analyse socio-économique de l'Internet, Paris, L'Harmattan. 
Retière, J.-N., 2003, « Autour de l'autochtonie. Réflexions sur la notion de capital social populaire », Politix, no 63, pp. 121-143.

Servais, C., 2017, « Scènes médiatiques et arènes de discours », Réseaux, no 202-203, pp. 79-121.

Schudson, M., 1997, «Why Conversation is Not the Soul of Democracy », Critical Studies in Mass Communication, vol. 14, pp. 297-309.

Smyrnaios, N., Marty, E., 2017, « Profession "Nettoyeur du Net” ", Réseaux, no 205, pp. 57-90.
Vignon, S., 2016, "Des maires en campagne(s). Transformations des répertoires de mobilisation électorale et des registres de légitimité politique dans les mondes ruraux ", Politix, no 113, pp. 17-42.

Voirol, O., 2017, " Pathologies de l'espace public et agitation fasciste : les "leçons" de la Théorie critique ", Réseaux, no 202-203, pp. 121-157. 


\title{
Resumé | Abstract | Résumo
}

\section{Commentaires dans la Presse Quotidienne Régionale française en ligne et politisation du local}

\author{
Comments in French Regional Online News and Local Politicization \\ Comentários no jornalismo regional on-line francês e politização do local
}

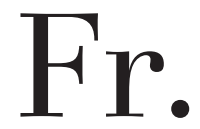

Cet article examine les commentaires postés sur le site d'un quotidien régional français, à la suite des articles consacrés à une commune moyenne dans la région toulousaine, durant la campagne municipale de 2014. Il s’attache à déterminer si des créations de communautés d'interprétation politique articulant la situation locale et une montée en généralité est observable et selon quelle logique elles s'organisent. Les commentaires sont analysés en les rapportant aux situations politiques et médiatiques locales et nationales et les commentateurs sont classés selon leurs prises de positions. Deux communautés sont finalement identifiées. La première, constituée de quelques membres maitrisant parfaitement la situation locale, peut être qualifiée d'autochtone ; elle interprète les faits et leur traitement par la presse selon une grille politique partisane correspondant à l'offre électorale du territoire. Elle est constituée de lecteurs intervenant quasi exclusivement sur la page de la commune. Ils participent plusieurs fois par semaine et sont régulièrement en conflit avec la ligne éditoriale du journal. La seconde communauté, plus nombreuse, n’intervient que sur les faits divers et se désintéresse de la situation locale. La montée en généralité est cependant également présente par l'intermédiaire de schémas interprétatifs proches de ceux imposés nationalement par l'extrême droite. Ces lecteurs interviennent sur tout le site du journal et de façon systématique dès qu'un événement peut laisser la place à une interprétation s'accordant à leur parti pris idéologique. Ils utilisent ainsi tous les faits divers pour proposer une politisation de la vie publique locale à travers un prisme marqué par le racisme, suggérant que tous les actes délictueux sont le fait de personnes d'origine étrangère et réclamant des sanctions de plus en plus radicales. Ils ne s'opposent pas à la ligne éditoriale du journal mais l'utilise en profitant de sa mise en avant systématique des faits divers. Ils contournent la modération par l'utilisation de para-phrases, de litotes, de métaphores ou de sous-entendus plus ou moins transparents et imposent ainsi au quotidien une vision du monde cohérente.

Mots-clés : Presse Quotidienne Régionale, publics, politique, participation, commentaires

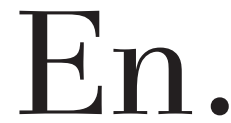

This paper analyzes comments posted on the website of a regional French daily serving a community near Toulouse in response to articles written during the 2014 municipal election campaign. The goal is to determine whether communities of local political discourse are being created and if they are, how are they organized and do generalities exist. Comments and their relationship with local and national politics and media are analyzed and commentators classified according to their position. Two distinct communities can be singled out: The first, consisting of a few commentators with an excellent grasp of the local situation, can be referred to as indigenous-they interpret events and how media handle them through a partisan regional lens. They write several times a week and are often at odds with the editorial line of the newspaper. The second, larger, community only comments on general events and has no interest in local situations. This increase in generality is abetted by interpretive models similar to those used by the far-right on the national level. These readers are active everywhere on the newspaper's website and systematically comment as soon as an event can be interpreted as being in line with their political ideology. In this way, they use local events to politicize local public life refracted through a racist lens in which all criminal acts are carried out by foreigners, and clamor for increasingly severe punishments. They do not oppose the newspaper's editorial line, but rather use it as a platform for their systematic commentary on local events. They 
circumvent judiciousness by paraphrasing and making use of euphemisms, metaphors and innuendo in varying degrees of transparency, and in so doing create a simulacrum of a coherent world.

Keywords: Daily regional press, publics, politics, participation, comments

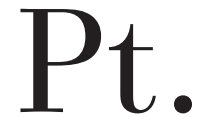

Este artigo examina os comentários postados no site de um jornal regional francês, produzidos logo após uma série de matérias sobre um distrito ("com- mune”) de porte médio, localizado na região de Toulouse, durante as eleições municipais de 2014. Busca determinar se os processos de criação de comunidades de interpretação política que articulam a situação local a um contexto mais generalizável podem ser observados, bem como o tipo de lógica na qual eles se organizam. Os comentários foram analisados a partir das relações entre as situações políticas e midiáticas locais e nacionais e a classificação dos comentaristas de acordo com os seus posicionamentos políticos. Assim, duas comunidades foram identificadas. A primeira, constituída de alguns integrantes que compreendiam perfeitamente a situação local, pode ser qualificada como autóctone; ela interpreta os fatos e o tratamento dado pela mídia de acordo com um quadro político partidário que corresponde à oferta eleitoral do território. Ela é constituída por leitores que postam comentários quase que exclusivamente na página dedicada à cobertura do distrito. Esses integrantes participam várias vezes por semana e frequentemente entram em conflito com a linha editorial do jornal. A segunda comunidade, mais numerosas, intervém apenas sobre os fait divers e não está interessada na situação local. O processo de generalização também está presente na análise por meio da utilização de esquemas interpretativos próximos àqueles impostos nacionalmente pela extrema direita. Esses leitores intervêm em todo o site do jornal e de forma sistemática, desde que um acontecimento dê abertura a uma interpretação que esteja em consonância com os seus posicionamentos ideológicos. Utilizam-se, assim, dos fait divers para propor uma politização da vida pública local sob o prisma do racismo, atribuindo a pessoas de origem estrangeira todos os fatos de caráter delituoso e reclamando sanções cada vez mais radicais contra essas pessoas. Eles não se opõem à linha editorial do jornal, utilizando-se dela em benefício de uma exposição sistemática dos fait divers. Para contornar a moderação, eles recorrem a paráfrases, lítotes, metáforas ou subentendidos mais ou menos transparentes, impondo ao jornal uma visão de mundo coerente.

Palavras-chave: Imprensa regional, públicos, política, participação, comentários

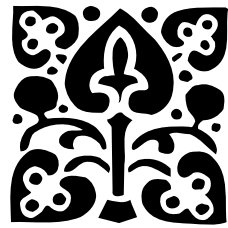

